\title{
Application of the Euler's gamma function to a problem related to F. Carlson's uniqueness theorem
}

\begin{abstract}
In his work on F. Carlson's uniqueness theorem for entire functions of exponential type, Q. I. Rahman [5] was led to consider an infinite integral and needed to determine the rate at which the integrand had to go to zero for the integral to converge. He had an estimate for it which he was content with, although it was not the best that could be done. In the present paper we find a result about the behaviour of the integrand at infinity, which is essentially best possible. Stirling's formula for the Euler's Gamma function plays an important role in its proof.
\end{abstract}

\section{Introduction.}

1.1. Carlson's theorem. Carlson's theorem says (see $[1$, Chapter 9]) that if $f$ is an entire function such that $|f(z)|=O\left(\mathrm{e}^{b|z|}\right)$ as $|z| \rightarrow \infty$ for some $b<\pi$ and $f(n)=0$ for $n=0, \pm 1, \pm 2, \ldots$, then $f(z)$ is identically zero. The example $f(z):=\sin \pi z$ shows that here $b=\pi$ is inadmissible. The following generalization of Carlson's theorem appears in [5] as Theorem 7.

Theorem A. Let $\left\{\lambda_{n}\right\}_{-\infty}^{\infty}$ be a sequence of real numbers such that $\left|n-\lambda_{n}\right| \leq$ $L$ for all $n \in \mathbb{Z}$ and $\left|\lambda_{n}-\lambda_{m}\right| \geq 2 \delta>0$ for $m \neq n$. Also, let $f(z)$ be an

2010 Mathematics Subject Classification. 32A15, 32A22.

Key words and phrases. Entire functions, Hadamard's three circles theorem, Euler's Gamma function. 
entire function and denote the maximum of $|f(z)|$ on $|z|=r$ by $M(r)$. Suppose that

$$
\int_{0}^{\infty} r^{2 Q} M(r) \mathrm{e}^{-\pi r} \mathrm{~d} r<\infty, \quad Q:=L+L / 2 \delta
$$

and that $f\left(\lambda_{n}\right)=0$ for all $n \in \mathbb{Z}$. Then $f(z) \equiv 0$.

The proof of Theorem A is, in part, based on the following auxiliary result presented in [5] as Lemma 6.

Proposition A. Let $M(r):=\max _{|z|=r}|f(z)|$, where $f$ is an entire function and let $Q$ be a positive number. Furthermore, let $\int_{0}^{\infty} r^{2 Q} M(r) \mathrm{e}^{-\pi r} \mathrm{~d} r<\infty$. Then, $r^{2 Q} M(r) \mathrm{e}^{-\pi r} \rightarrow 0$ as $r \rightarrow \infty$.

Unless $f$ is a constant, $M(r)$ is an increasing function of $r$. This is all that was needed in the proof of Proposition 1, as given in [5]. Here we prove a result (Theorem 1) from which it follows that

$$
r^{2 Q+1 / 2} M(r) \mathrm{e}^{-\pi r}=O(1) \text { as } r \rightarrow \infty .
$$

Our approach to the problem is different. We relate it to Euler's Gamma function and make effective use of Stirling's formula to obtain the result. In our proof of Theorem 1 we also use the fact that $\log M(r)$ is a convex function of $\log r$ and not simply a non-decreasing function $r$. Our proof clearly suggests how to prove that (1.2) is best possible, as far as the number $1 / 2$ in $r^{2 Q+1 / 2}$ goes.

Theorem 1. Let $M(r)=M(r, f):=\max _{|z|=r}|f(z)|$, where $f$ is an entire function and suppose that $\int_{0}^{\infty} r^{\alpha} M(r) \mathrm{e}^{-\beta r} \mathrm{~d} r<\infty$ for some $\alpha>0$ and some $\beta>0$. Then $r^{\alpha+1 / 2} M(r) \mathrm{e}^{-\beta r}=O(1)$ as $r \rightarrow \infty$.

\section{Some facts about $M(r)$ and the Stirling's formula.}

2.1. Convexity of $\log M(r)$ as a function of $\log r$. Hadamard's threecircles theorem [6, p. 172] says: Let $f(z)$ be an analytic function, regular for $r_{1} \leq|z| \leq r_{3}$. Furthermore, let $r_{1}<r_{2}<r_{3}$, and let $M_{1}, M_{2}, M_{3}$ be the maxima of $|f(z)|$ on the three circles $|z|=r_{1}, r_{2}, r_{3}$, respectively. Then

$$
M_{2}^{\log \left(r_{3} / r_{1}\right)} \leq M_{1}^{\log \left(r_{3} / r_{2}\right)} M_{3}^{\log \left(r_{2} / r_{1}\right)} .
$$

Since we may write $(2.1)$ in the form

$$
\log M\left(r_{2}\right) \leq \frac{\log r_{3}-\log r_{2}}{\log r_{3}-\log r_{1}} \log M\left(r_{1}\right)+\frac{\log r_{2}-\log r_{1}}{\log r_{3}-\log r_{1}} \log M\left(r_{3}\right),
$$

Hadamard's three-circles theorem may be expressed by saying that $\log M(r)$ is a convex function of $\log r$.

In our case, $M(r):=\max _{|z|=r}|f(z)|$, where $f$ is an entire function. Unless $f$ is a constant, $M(r)$ is a strictly increasing function of $r$. It is easily seen that $f(z)$ is a polynomial of degree $n$, that is $f(z):=\sum_{\nu=0}^{n} a_{\nu} z^{\nu}, a_{n} \neq 0$ if 
and only if $\log M(r) /(\log r) \rightarrow n$ as $r \rightarrow \infty$. From (2.2) it follows that if $f(z)$ is a transcendental entire function, then there exists a number $r_{0}$ such that $\log M(r) /(\log r)$ is an unbounded strictly increasing function of $r$ for $r \geq r_{0}$.

We know that $\log M(r)$ is continuous. In addition, it is a convex function of $\log r$. It is known (see [2, p. 142]) that a continuous convex function has finite right-hand and left-hand derivatives at each point, and that these derivatives themselves are nondecreasing functions.

2.2. Stirling's formula. Our proof of Theorem 1 uses Stirling's formula for the Gamma function defined by the Eulerian integral of the second kind $\Gamma(z)=\int_{0}^{\infty} \mathrm{e}^{-t} t^{z-1} \mathrm{~d} t$ whenever this integral converges (it being understood that $t^{z-1}$ has its principal value), and defined by analytical continuation elsewhere. Stirling's formula says [4, p. 42] that

$$
\Gamma(z)=\sqrt{2 \pi} z^{z-1 / 2} \mathrm{e}^{-z} \mathrm{e}^{J(z)},
$$

where the power of $z$ has its principal value and

$$
0<J(x)<1 /(12 x) \quad(x>0) .
$$

3. Proof of Theorem 1. Setting $\beta r=u$, we see that

$$
\int_{0}^{\infty} r^{\alpha} M(r, f) \mathrm{e}^{-\beta r} \mathrm{~d} r=\frac{1}{\beta^{\alpha+1}} \int_{0}^{\infty} u^{\alpha} M(u, g) \mathrm{e}^{-u} \mathrm{~d} u,
$$

where $g(z):=f(z / \beta)$ is an entire function. It is therefore enough to prove Theorem 1 in the special case where $\beta=1$. Thus, we have to prove that if $f(z)$ is an entire function such that

$$
\int_{0}^{\infty} r^{\alpha} M(r, f) \mathrm{e}^{-r} \mathrm{~d} r<\infty
$$

for some $\alpha>0$, then

$$
r^{\alpha+1 / 2} M(r, f) \mathrm{e}^{-r}=O(1) \text { as } r \rightarrow \infty .
$$

The result is trivial if $f(z)$ is a polynomial. So, let $f(z)$ be a transcendental entire function. By considering $F(z):=f(z)-f(0)+1$ if necessary, we may suppose that $\log M(r, f)$ is a positive increasing convex function of $\log r$ in $-\infty<\log r<\infty$. Note that (3.1) holds if and only if

$$
\int_{0}^{\infty} r^{\alpha} M(r, F) \mathrm{e}^{-r} \mathrm{~d} r<\infty
$$

and that (3.2) holds if and only if $r^{\alpha+1 / 2} M(r, F) \mathrm{e}^{-r}=O(1)$ as $r \rightarrow \infty$.

Hereafter we shall simply write $M(r)$ for $M(r, f)$ and $M(s)$ for $M(s, f)$ because we see no confusion in doing so. 
In view of all that has been said in $\S 2.1$, for any $s>0$ there is a constant $C=C(s)$ such that

$$
\log M(r) \geq \log M(s)+C \log (r / s) \quad(r>0),
$$

and that there is an $s_{0}$ such that $C(s) \geq 1$ for all $s \geq s_{0}$. Hence, for any $s \geq S_{0}:=\max \left\{s_{0}, 10\right\}$, we have

$$
\begin{aligned}
A & :=\int_{0}^{\infty} r^{\alpha} M(r) \mathrm{e}^{-r} \mathrm{~d} r \geq M(s) \int_{0}^{\infty} r^{\alpha}(r / s)^{C} \mathrm{e}^{-r} \mathrm{~d} r \\
& =M(s) s^{-C} \Gamma(C+\alpha+1) .
\end{aligned}
$$

Taking (2.4) into account, it follows from (2.3) that

$$
\Gamma(C+\alpha+1)>\frac{\sqrt{2 \pi}}{\mathrm{e}}(C+\alpha)^{C+\alpha+1 / 2} \mathrm{e}^{-(C+\alpha)},
$$

and so

$$
s^{\alpha} M(s)<A \frac{\mathrm{e}}{\sqrt{2 \pi}} \mathrm{e}^{C+\alpha} s^{C+\alpha} \frac{1}{(C+\alpha)^{C+\alpha+1 / 2}} .
$$

Thus we see that

$$
s^{\alpha} M(s)<A \frac{\mathrm{e}}{\sqrt{2 \pi}} \max _{t \geq 1}\left\{(\mathrm{e} s / t)^{t} t^{-1 / 2}\right\} .
$$

Let us define

$$
\varphi(t):=(\mathrm{e} s / t)^{t} t^{-1 / 2} .
$$

In order to obtain a good upper estimate for $\max _{t \geq 1} \varphi(t)$ we note that $\varphi^{\prime}(t)=0$ if and only if

$$
B(t):=\log s-\left(\log t+\frac{1}{2 t}\right)=0 .
$$

From this it is easily seen that $\varphi^{\prime}(t)$ has one and only one zero in $(1, \infty)$. Let us call it $\tau_{s}$. We claim that

$$
s-\frac{1}{2}-\frac{1}{s-(1 / 2)} \leq \tau_{s} \leq s-\frac{1}{2} \quad\left(s>S_{0}\right) .
$$


For this, note that

$$
\begin{aligned}
B\left(s-\frac{1}{2}\right. & \left.-\frac{1}{s-(1 / 2)}\right)=\log \frac{s\left(s-\frac{1}{2}\right)}{\left(s-\frac{1}{2}\right)^{2}-1}-\frac{1}{2} \frac{s-\frac{1}{2}}{\left(s-\frac{1}{2}\right)^{2}-1} \\
& =\log \left\{1+\frac{\frac{1}{2}\left(s-\frac{1}{2}\right)+1}{\left(s-\frac{1}{2}\right)^{2}-1}\right\}-\frac{1}{2} \frac{s-\frac{1}{2}}{\left(s-\frac{1}{2}\right)^{2}-1} \\
& >\frac{\frac{1}{2}\left(s-\frac{1}{2}\right)+1}{\left(s-\frac{1}{2}\right)^{2}-1}-\frac{1}{2} \frac{\frac{1}{4}\left(s-\frac{1}{2}\right)^{2}+s+\frac{1}{2}}{\left\{\left(s-\frac{1}{2}\right)^{2}-1\right\}^{2}}-\frac{\frac{1}{2}\left(s-\frac{1}{2}\right)}{\left(s-\frac{1}{2}\right)^{2}-1} \\
& =\frac{\frac{7}{4}\left(s-\frac{1}{2}\right)^{2}-\left(s-\frac{1}{2}\right)-3}{2\left\{\left(s-\frac{1}{2}\right)^{2}-1\right\}^{2}}>0,
\end{aligned}
$$

whereas

$$
B\left(s-\frac{1}{2}\right)=\log \frac{s}{s-(1 / 2)}-\frac{1}{2 s-1}=\log \left(1+\frac{1}{2 s-1}\right)-\frac{1}{2 s-1}<0 .
$$

It follows that if $\varphi(t)$ is as in (3.4) then

$$
\begin{aligned}
\max _{t \geq 1} \varphi(t) & =\max \left\{t^{-1 / 2} \mathrm{e}^{t}(s / t)^{t}: s-\frac{1}{2}-\frac{1}{s-(1 / 2)} \leq t \leq s-\frac{1}{2}\right\} \\
& \sim s^{-1 / 2} \mathrm{e}^{s} \text { as } s \rightarrow \infty
\end{aligned}
$$

since

$$
t^{-1 / 2} \sim s^{-1 / 2}, \mathrm{e}^{t} \sim \mathrm{e}^{-1 / 2} \mathrm{e}^{s} \text { and }(s / t)^{t} \rightarrow \mathrm{e}^{1 / 2} \text { as } s \rightarrow \infty .
$$

Using this fact about $\max _{t \geq 1} \varphi(t)$ in (3.3), we obtain the desired result.

Remark 1. The proof of Theorem 1 is of a somewhat wider scope than it might appear. In fact, the property of the function $M(r)$ by which $\log M(r)$ is a convex function of $\log r$ is shared by some other functions associated with an entire function $f$. For example, if

$$
\mathfrak{M}_{p}(r):=\left(\frac{1}{2 \pi} \int_{0}^{2 \pi}\left|f\left(r \mathrm{e}^{\mathrm{i} \theta}\right)\right|^{p} \mathrm{~d} \theta\right)^{1 / p}, p>0,
$$

then, $\log \mathfrak{M}_{p}(r)$ is a convex function of $\log r$ for any $p>0$. This is a wellknown result of G. H. Hardy [3]. If $f(z):=\sum_{n=0}^{\infty} a_{n} z^{n}$, then for any $r>0$, the maximum of $\left|a_{n}\right| r^{n}$ for $n \in\{0,1,2, \ldots\}$ is called the maximum term. It is usually denoted by $\mu(r)$ and $\log \mu(r)$ is known [7, pp. 30-31] to be a convex function of $\log r$.

Remark 2. Infinite integrals arise in various areas of pure and applied mathematics as well as in Statistics. They are also of interest to physicists and engineers. So, a result like Theorem 1 has the potential to be useful in the future. As an immediate application of the result, we state the following 
generalization of Theorem A where condition (1.1) has been replaced by a less restrictive one.

Corollary 1. Let $\left\{\lambda_{n}\right\}_{-\infty}^{\infty}, L$ and $Q$ be as in Theorem A. Also, let $f(z)$ be an entire function and denote the maximum of $|f(z)|$ on $|z|=r$ by $M(r)$. Suppose that $\int_{0}^{\infty} r^{2 Q-\gamma} M(r) \mathrm{e}^{-\pi r} \mathrm{~d} r<\infty$ for some $\gamma<1 / 2$ and that $f\left(\lambda_{n}\right)=0$ for all $n \in \mathbb{Z}$. Then $f(z) \equiv 0$.

The proof of Corollary 1 requires only a minor modification in the proof of Theorem A, as given in [7], and so we omit it.

\section{REFERENCES}

[1] Boas, Jr., R. P., Entire Functions, Academic Press, New York, 1954.

[2] Boas, Jr., R. P., A Primer of Real Functions, The Carus mathematical monographs, No. 13, The Mathematical Association of America, 1960.

[3] Hardy, G. H., The mean value of the modulus of an analytic function, Proc. London Math. Soc. 14 (1915), 269-277.

[4] Henrici, P., Applied and Computational Complex Analysis, Vol. 2, (A WileyInterscience publication), John Wiley \& Sons, New York, 1977.

[5] Rahman, Q. I., Interpolation of Entire functions, Amer. J. Math. 87 (1965), 10291076.

[6] Titchmarsh, E. C., The Theory of Functions, 2nd ed. Oxford University Press, 1939.

[7] Valiron, G., Lectures on the General Theory of Integral Functions, Chelsea Publishing Company, New York, 1949.

M. A. Qazi

Department of Mathematics

Tuskegee University

Tuskegee, AL 36088

U.S.A.

e-mail: qazima@aol.com

Received January 16, 2016 